\title{
Efficient Relay Selection Algorithm for Non-Orthogonal Amplify-and-Forward Cooperative Systems over Block-Fading Channels
}

\author{
Yunida YUNIDA, Rusdha MUHARAR, Yuwaldi AWAY, Nasaruddin NASARUDDIN \\ Dept. of Electrical and Computer Engineering, Universitas Syiah Kuala, Banda Aceh, Indonesia \\ nasaruddin@unsyiah.ac.id
}

Submitted September 28, 2019 / Accepted March 3, 2020

\begin{abstract}
In this paper, an efficient relay selection (RS) algorithm for non-orthogonal amplify-and-forward (NAF) cooperative systems over block-fading channels, also known as block-fading NAF (BFNAF) protocol, is developed. The best relay is selected from a subset of available relay nodes based on the maximum criterion of their capacity bounds in half-duplex (HD) mode, together with the power allocation, to obtain the energy efficiency (EE) for the proposed RS scheme. We derived an exact closed-form expression of the outage probability and throughput for evaluating the system performance. The energy consumption was also numerically evaluated to determine the optimized EE of the proposed RS scheme for each transmission protocol with two modulation schemes. The numerical results indicated that the proposed $R S$ scheme with the $B F N A F$ protocol outperforms the previous $R S$ scheme with orthogonal AF (OAF) protocol in terms of both the outage probability and the throughput as the number of relays is increased and the average transmit power is optimally allocated for each transmission phase. Moreover, in the case of the optimized EE, it is found that by using quadrature amplitude modulation (QAM), the EE of the proposed $R S$ scheme is $48.9 \%$ higher than that of binary phase-shift keying (BPSK) modulation.
\end{abstract}

\section{Keywords}

Block-fading, capacity bound, efficient relay selection, energy efficiency, non-orthogonal amplifyand-forward, power allocation

\section{Introduction}

Cooperative networks in the wireless channel have been proven to increase the data rate and coverage area, and reduce the transmission power constraint in the communication process [1]. Generally, a better performance gain of cooperative systems can be obtained by relaying protocols such as decode-and-forward (DF) [2-4], and amplify-and-forward (AF) in [2, 5-7]. The DF protocol is more complicated than the $\mathrm{AF}$ protocol because the relay nodes must first decode the received signal from a source node and then forward the re-encoded signal to the destination. In contrast, the AF protocol is more straightforward because the relay node only needs to amplify and re-transmit the amplified version of the information signal without other signal processing. The AF protocol, also called orthogonal AF (OAF), was first introduced by Laneman et al. [2], in which time-division multiplexing (TDM) is used to split the transmission process into two phases. The first phase is called the broadcasting phase, where the source node transmits the information signal to the relay and the destination. The second phase is the cooperative phase, where the relay node re-transmits the amplified version of the information signal to the destination. However, this approach causes a loss of the data rate (bandwidth) as a result of an inactive source node in the second phase.

To overcome this limitation, Nabar et al. [8] proposed a new AF protocol for half-duplex cooperative networks, called the non-orthogonal AF (NAF) protocol, where the source continuously transmits a new information signal during the second phase. By using the NAF protocol, the broadcast range and the received signal-to-noise ratio (SNR) can be maximized [9] and the relay nodes can transmit during both transmission phases simultaneously. For the block-fading channel scenario where the channel condition is changed during the second phase, Krikidis et al. [10] proposed another NAF-based protocol, called the block-fading NAF (BFNAF), which is compatible for low spectral efficiency. The BFNAF protocol has a similar transmission process to that of the classic NAF, except that the source re-transmits the same information signal during the second phase to increase the diversity gain.

The improvement of the diversity gain in distributed networks such as cooperative and multiple-input multipleoutput (MIMO) systems can be achieved by orthogonal transmission, pre-coding, maximum ratio combining (MRC), or distributed space-time coding (STC). The orthogonal transmission has low spectral efficiency and suffers from a rate loss or bandwidth penalty [4]. In addition, with pre-coding design [11] and STC [12], the system needs exact channel state information (CSI) and precise symbol-level synchronization, which is hard to obtain. 
In this research, a selection process is proposed as a practical and efficient method to solve the above-mentioned problems by selecting the "best" antenna in the MIMO systems and the "best" relay in cooperative networks. In our previous work [11], we introduced a NAF relaying scheme for the cooperative MIMO system by designing the linear pre-coding to increase the transmit gain by using the minimum mean square error (MMSE) criterion in HD mode. The numerical result shows that the proposed pre-coded scheme outperforms the un-pre-coded and the zero-forcing (ZF) pre-coded-based schemes. For cooperative networks, the relay selection method uses the limited power and bandwidth resource efficiently. A relaying scheme that prevents the excessive use of resources is called opportunistic relay selection (ORS) or best relay selection (BRS) [13], [14]. In ORS, the source either broadcasts or encourages one of the available relay nodes to receive the signal via a selection method that contains the trade of CSI between the relay nodes and the transceiver pairs.

Krikidis et al. [15] proposed a relay selection scheme for full-duplex (FD) OAF relaying networks by using both global and partial CSI. The relay selection strategies are analyzed in terms of outage probability using a closed-form high SNR that enables better performance gain. However, due to the residual loop interference at the relay, FD relaying results in zero diversity order despite the relay selection process. Nevertheless, despite the advantage of FD relaying systems, the complexity level in the context of multiple relays and RS strategies still becomes the main problem. Due to the existence of the NAF relaying, which is considered as degeneration of linear relaying to FD operation in [16], the extra residual self-interference can be omitted.

The performance of non-orthogonal single relay cooperative systems is degraded by the existence of direct transmission in the second phase. Therefore, an efficient technique has recently been proposed for NAF relaying systems in underlay spectrum sharing networks [17]. Elsaadany et al. solved the problem by using power allocation as the energy efficiency (EE) technique to increase the performance gain of a cooperative network. The EE design for a combined RS and power allocation of the OAF systems has been studied in [18], where the EE maximization design is obtained by optimizing the minimum data rate and the maximum transmitted power for each node.

An efficient RS algorithm for orthogonal cooperative networks has been proposed in [19], using the idea of the multi-branch switched diversity system called the "switchand-examine node selection (SENS)" algorithm. The proposed scheme provides much lower complexity and channel estimation, which results in reduced energy consumption at the relay node. The error performance of this system was similar to that of the ORS scheme in the low SNR regime, but lower in the high SNR regime. Do \& Le in [20] introduced a new non-orthogonal multiple-access (NOMA) scheme based cognitive AF relaying, in order to increase the performance gain and the EE by means of employing the ORS and partial relay selection (PRS) scheme under hardware impairments. The outage performances show that NOMA provided a better performance gain compared to that of the orthogonal case. As for the EE, the ORS scheme is more efficient than that of the PRS schemes due to the increasing function of transmitted power at the source node. To the best of our knowledge, an efficient RS scheme for the cooperative system with BFNAF protocol has not been investigated previously.

In this paper, we propose an efficient RS algorithm for non-orthogonal HD cooperative networks with the BFNAF protocol over block-fading channels. We consider the BFNAF protocol in cooperative systems with a new relay selection method. The BFNAF protocol, introduced in [10], can increase the performance of a single relay system besides using the NAF protocol that has been a focus of cooperative protocol in our previous works. Furthermore, Krikidis et al. [10] also suggested some ideas for future works to consider a multi-relay system using a relay selection scheme to achieve better performance. Hence, based on this motivation, we proposed a new relay selection algorithm based on the capacity bound of source-torelay and relay-to-destination links for the multi-relay system by using the BFNAF protocol as a cooperative transmission that has not yet been proposed in open-access literature.

The relay is selected by maximizing the capacity bound of the available relay nodes. Performance analysis in terms of outage probability and throughput for this proposed scheme was carried out. The numerical results show that the proposed scheme has a better channel capacity compared to that of the OAF protocol and outperforms the OAF both with and without the relay selection method. The outage performance and the throughput of the proposed scheme can be significantly improved by increasing the numbers of relay nodes and/or by allocating the average transmit power of the relay node optimally as a part of the amplification process. In addition, an EE analysis was also performed to determine the energy consumption of the proposed scheme with the BFNAF and OAF protocol. Based on the simulation results, the EE of the proposed scheme is $85.80 \%$ for $M$-quadrature amplitude modulation (16-QAM) and $36.9 \%$ for binary phase-shift keying (BPSK) with the amount of the total available power transmission.

The main contributions of this paper are:

1) An efficient RS algorithm for a BFNAF protocol in multi-relay HD cooperative networks based on the maximum criterion of the capacity bounds of relay nodes.

2) The outage and throughput performance of the BFNAF protocol based on the upper-bound capacity of the source-to-relay and relay-to-destination links for different numbers of relays.

3) An analysis of the energy consumption of the proposed RS algorithm with additional optimal power allocation for the BFNAF protocol to determine the optimized EE in the HD cooperative system. 
The remaining parts of this work are formatted as follows. The system model and the proposed RS algorithm for a non-orthogonal HD cooperative network with the BFNAF protocol are presented in Sec. 2 and Sec. 3, respectively. The outage and throughput and the $\mathrm{EE}$ analysis are investigated in Sec. 4 and Sec. 5, respectively. The numerical results for capacity, outage performance, throughput, and EE are presented in Sec. 6. Finally, a brief conclusion is presented in Sec. 7 .

\section{System Model}

In this section, we present a system model of the HD cooperative networks with NAF relaying over block-fading channels. The system model for the non-orthogonal relaying network with multiple relays consists of one source, $\mathrm{S}$, one destination, $\mathrm{D}$, and a number of $K$ relay nodes, with $\mathbf{R}=\left\{\mathrm{R}_{1}, \mathrm{R}_{2}, \ldots, \mathrm{R}_{K}\right\}$, each equipped with a single antenna as illustrated in Fig. 1. The communication process is divided into two transmission phases: the broadcasting and cooperating phases, respectively. In the first phase, $\mathrm{S}$ transmits the information signal with an average transmit power $P_{\mathrm{S}}{ }^{(1)}$ to $\mathrm{D}$ and all $K$ relay nodes, using the considered modulation schemes. The $k$-th relay, denoted by $\mathrm{R}_{k}$, is selected out from $K$ relay nodes, and then amplifies the information signal in the analog domain and retransmits it with an average transmit power $P_{\mathrm{R}_{k}}$ to $\mathrm{D}$ in the second phase. For non-orthogonal transmission, $\mathrm{S}$ also retransmits the information signal to $\mathrm{D}$ with an average transmit power of $P_{\mathrm{S}}^{(2)}$.

We assume that the channel between every two of the three nodes is Rayleigh block flat-fading with bandwidth $B$ $[\mathrm{Hz}]$ that keeps varying during each transmission phase. The signal received at $\mathrm{D}$ and the $k$-th relay, respectively, can be written as

$$
\begin{aligned}
& y_{\mathrm{D}}^{(1)}=\sqrt{P_{\mathrm{S}}^{(1)}} h_{\mathrm{SD}}^{(1)} x_{1}+n_{\mathrm{D}}^{(1)}, \\
& y_{\mathrm{R}_{k}}^{(1)}=\sqrt{P_{\mathrm{S}}^{(1)}} h_{\mathrm{SR}_{k}}^{(1)} x_{1}+n_{\mathrm{R}_{k}}^{(1)}
\end{aligned}
$$

where $y_{\mathrm{D}}^{(1)}$ and $y_{\mathrm{R}_{k}}^{(1)}$ denote the signals received at $\mathrm{D}$ and the $k$-th relay, $\mathrm{R}_{k}$, where $k=1,2, \ldots, K$, during the first phase, respectively. $x_{1}$ denotes the transmitted signal in the first phase with an average transmit power $\mathrm{E}\left(x_{1} x_{1}{ }^{*}\right)=1$. $h_{\mathrm{SD}}{ }^{(1)}$ and $h_{\mathrm{SR}_{k}}{ }^{(1)}$ denote the channel coefficients for links $\mathrm{S} \rightarrow \mathrm{D}$ and $\mathrm{S} \rightarrow \mathrm{R}_{k}$ in the first phase, respectively. $n_{\mathrm{D}}{ }^{(1)}$ and $n_{\mathrm{R}_{k}}^{(1)}$ are the additive white Gaussian noises (AWGN) at D and $\mathrm{R}_{k}$ in the first phase, respectively. For simplicity, all of the noise vectors are assumed to have the same variance $\sigma^{2}$.

After the relay selection process, discussed in the next section, the signal received at the selected $k$-th relay, $\mathrm{R}_{k}$, is multiplied by an amplification factor $\beta$, which can be written as [4]

$$
x_{\mathrm{R}_{k}}=\beta y_{\mathrm{R}_{k}}^{(1)},
$$

with $\beta=\sqrt{P_{\mathrm{R}_{k}} /\left(P_{\mathrm{S}}^{(1)}\left|h_{\mathrm{SR}_{k}}^{(1)}\right|+\sigma^{2}\right)}$.

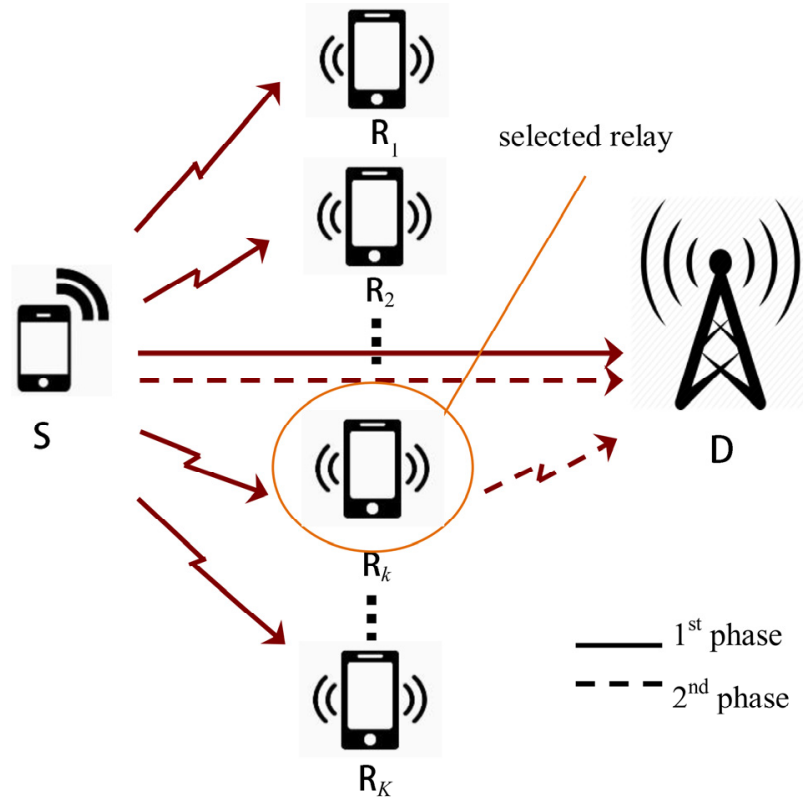

Fig. 1. A system model of a non-orthogonal half-duplex cooperative network with multiple relays.

Furthermore, by assuming that the system operates in non-orthogonal HD transmission mode, $\mathrm{S}$ continues to transmit the information signal, and the selected relay $\mathrm{R}_{k}$ simultaneously forwards the amplified signal defined by (3) to D in the second phase.

Thus, in this phase, D receives signal vectors both from $\mathrm{S}$ and from the selected relay $\mathrm{R}_{k}$ written as follows:

$$
y_{\mathrm{D}}^{(2)}=\sqrt{P_{\mathrm{S}}^{(2)}} h_{\mathrm{SD}}^{(2)} x_{2}+\sqrt{P_{\mathrm{R}_{k}}} h_{\mathrm{R}_{k} \mathrm{D}}^{(2)}\left(\beta y_{\mathrm{R}_{k}}^{(1)}\right)+n_{\mathrm{D}}^{(2)}
$$

where $h_{\mathrm{SD}}{ }^{(2)}$ and $h_{\mathrm{R}_{k} \mathrm{D}}{ }^{(2)}$ denote the channel coefficients for the direct link and the link $\mathrm{R}_{k} \rightarrow \mathrm{D}$ in the second phase, respectively. $x_{2}$ denotes the new transmitted symbol in the second phase with an average transmit power of $\mathrm{E}\left(x_{2} x_{2}{ }^{*}\right)=1$, and $n_{\mathrm{D}}{ }^{(2)}$ is the power of the noise term at $\mathrm{D}$ in the second phase with the variance $\sigma^{2}$.

As well as the NAF relaying, the transmission process for the OAF scheme is also performed in two phases. For the first phase, $\mathrm{S}$ transmits its information signal with transmit power $P_{\mathrm{S}}^{(1)}$ to $\mathrm{R}$ and $\mathrm{D}$ simultaneously. The selected relay $\mathrm{R}_{k}$ then amplifies and forwards the noisy version of its signal received from $\mathrm{S}$ to $\mathrm{D}$ during the second phase. In other words, during the second phase only the selected relay is active in the transmission process while $\mathrm{S}$ is inactive; $\left(P_{\mathrm{S}}^{(2)}=0\right)$. However, the NAF protocol has been proven by Elsaadany \& Hamouda in [9] as an optimal scheme for HD single relay channels in terms of diversitymultiplexing-tradeoff (DMT) compared to the OAF. In particular, to prevent the data transmission rate loss which appears from the idleness of the $\mathrm{S}$ during the second phase for the OAF protocol, the classic NAF protocol permits the $\mathrm{S}$ to also participate in direct transmission during the second phase. This feature arises from the fact that the $\mathrm{S}$ continuously maintains the transmission of new data $\left(x_{2}\right)$ while the relay node retransmits the amplified version of the data signal from the source node $\left(x_{1}\right), x_{1} \neq x_{2}$. 
The advanced study of the cooperative protocols for the quasi-static block-fading channels resulted in another new NAF-based protocol called block-fading NAF (BFNAF). This new cooperative protocol provides an extra diversity order and permits the $S$ of the classic NAF protocol to retransmit the same information signal during the second phase to improve the diversity gain at the $D$. We also assume that the channels are the block flat-fading that keep invariant during the cooperative phase $\left(h_{\mathrm{SD}}^{(1)} \neq h_{\mathrm{SD}}^{(2)}\right)$. Thus, the information signals transmitted during the first phase indicated in (1) and the second phase indicated in (4) are equal $\left(x_{1}=x_{2}=x\right)$. Therefore, the total received signal at $\mathrm{D}$ during both transmission phases can be expressed as:

$\left[\begin{array}{c}y_{\mathrm{D}}^{(1)} \\ y_{\mathrm{D}}^{(2)}\end{array}\right]=\left[\begin{array}{cc}\sqrt{P_{\mathrm{S}}^{(1)}} h_{\mathrm{SD}}^{(1)} & 0 \\ \frac{\sqrt{P_{\mathrm{S}}^{(1)} P_{\mathrm{R}_{k}}}}{\sqrt{1+P_{\mathrm{R}_{k}} \beta^{2}\left|h_{\mathrm{R}_{k} \mathrm{D}}^{(2)}\right|^{2}}} \beta h_{\mathrm{SR}_{k}}^{(1)} h_{\mathrm{R}_{k} \mathrm{D}}^{(2)} & \frac{\sqrt{P_{\mathrm{S}}^{(2)} h_{\mathrm{SD}}^{(2)}}}{\sqrt{1+P_{\mathrm{R}_{k}} \beta^{2}\left|h_{\mathrm{R}_{k} \mathrm{D} \mid}^{(2)}\right|^{2}}}\end{array}\right] \cdot\left[\begin{array}{l}x \\ x\end{array}\right]+\mathbf{Z}$

where $\mathbf{Z}=\left[\begin{array}{c}n_{\mathrm{D}}^{(1)} \\ \beta h_{\mathrm{SR}_{k}}^{(1)} n_{\mathrm{R}_{k}}^{(1)}+n_{\mathrm{D}}^{(2)}\end{array}\right]$ is the noise vector at $\mathrm{D}$.

\section{Proposed Relay Selection Algorithm}

In this section, we investigate a new relay selection algorithm for the non-orthogonal cooperative protocol using the maximum criterion of the capacity bounds of the relay nodes. Then, we present the application of the ORS scheme for non-orthogonal protocol based on the maximum-minimum criterion of the end-to-end received signalto-noise ratio (SNR) of source and relay nodes.

\subsection{Opportunistic Relay Selection}

The ORS method in [13], [14] offers the best performance gain with low complexity and is considered the most proportional scheme for non-orthogonal transmission cases such as the NAF and BFNAF relaying systems. However, the continuous transmission in non-orthogonal relaying does not always provide the best performance. In poor channel conditions, these relaying processes also greatly amplify the noise variable, which could result in a degradation of the performance of the direct transmission. To prevent such deficiency in the ORS as the best solution, the selected relay $\mathrm{R}_{k}$ is the relay node with the best end-toend received SNR related to its average transmit power written as [13]

$$
\mathrm{R}_{k_{-} \mathrm{ORS}}=\arg \max _{k=1,2, \ldots .}\left\{\min \left(\mathrm{SNR}_{\mathrm{SR}_{k}}^{(1)}, \mathrm{SNR}_{\mathrm{R}_{k} \mathrm{D}}^{(2)}\right)\right\}
$$

where $\mathrm{SNR}_{\mathrm{SR}_{k}}^{(1)}=\gamma_{\mathrm{SR}_{k}}^{(1)}=\frac{P_{\mathrm{S}}^{(1)}}{N_{0}}\left|h_{\mathrm{SR}_{k}}^{(1)}\right|^{2}$ is the instantaneous endto-end received SNR for link $\mathrm{S} \rightarrow \mathrm{R}_{k}$ in the first phase and $\mathrm{SNR}_{\mathrm{R}_{k} \mathrm{D}}^{(2)}=\frac{\gamma_{\mathrm{SR}_{k}}^{(1)} \gamma_{\mathrm{R}_{k} \mathrm{D}}^{(2)}}{\gamma_{\mathrm{SR}_{k}}^{(1)}+\gamma_{\mathrm{R}_{k} \mathrm{D}}^{(2)}+1}$ is the instantaneous end-to-end received SNR in the second phase, where $\gamma_{\mathrm{R}_{k} \mathrm{D}}^{(2)}=\frac{P_{\mathrm{R}_{k}}}{N_{0}}\left|h_{\mathrm{R}_{k} \mathrm{D}}^{(1)}\right|^{2}$ is the end-to-end received SNR for link $\mathrm{R}_{k} \rightarrow \mathrm{D}$.

Based on (6), we can see that the selection method for the ORS scheme is related to the exchange of the channel condition or CSI between the source and the relay node. To achieve a better performance gain, both source and relay must have a perfect knowledge of the CSI to allow the retransmission process of the information signal to the destination. Moreover, the ORS scheme also requires continuous channel feedback from all available links, which causes high energy consumption. Therefore, we proposed a new RS scheme which uses the channel capacity bound as the measurement of the channel condition and implies the optimal power allocation scheme for EE purposes for non-orthogonal cooperative networks.

\subsection{Proposed Relay Selection}

The EE has recently been an interesting issue in cooperative research areas [17-20], and is the solution for the limited resource problem by using the broadcast nature of the wireless channel. In this work, we propose an efficient RS scheme for the BFNAF protocol in HD cooperative networks based on the channel capacity bounds of the source-to-relay and relay-to-destination links.

The new proposed RS algorithm for the BFNAF relaying system can be described as the three following steps:

1) In the first step a subset of available relay nodes $(K)$, which can overhear each other and the destination, is initialized and maintained in listening mode during the transmission process.

2) Calculate the instantaneous end-to-end received SNR at the $k$-th relay nodes during the first phases based on (6). In this work, we proposed an efficient RS scheme where the instantaneous capacity $\left(C_{\mathrm{R}_{K_{-}} \text {coop }}\right)$ of available relay nodes for link $\mathrm{S} \rightarrow \mathrm{R}_{k}$ can be used to facilitate the selection process by applying maximum-ratio combining (MRC) given by

$$
C_{\mathrm{R}_{K_{-}} \text {coop }}=\min \left\{R_{0}, \frac{1}{2} \log _{2}\left(1+\mathrm{SNR}_{\mathrm{SR}_{K}}^{(1)}\right)\right\}
$$

where $R_{0}$ is the desired data transmission rate of the systems in bits per second (bps) and the factor of $1 / 2$ corresponds to the two transmission phases.

3) Based on (7), for the case where the capacity of each relay node is lower than the desired data transmission rate $\left(C_{\mathrm{R}_{K_{-}} \text {coop }}<R_{0}\right)$, the relay is selected based on its instantaneous received SNR maximum during the first and second phases (ORS scheme). On the other hand, if the capacities of the relay nodes are higher than the desired data transmission rate $\left(C_{\mathrm{R}_{K} \text { coop }}>R_{0}\right)$, the best relay is selected with the maximum capacity bound among all of the available relay nodes. The best relay, $\mathrm{R}_{k}$, is selected based on the maximum 
criterion of the capacity bounds, which can be expressed as follows:

$$
\mathrm{R}_{k}=\arg \max _{k=1,2, . . K}\left\{C_{\mathrm{R}_{k-} \text { coop }}\right\} \text {. }
$$

Given the instantaneous capacity of the selected relay in (7), the proposed RS scheme still requires an optimization scheme that can simplify the complexity of the system analysis. The instantaneous capacity for each of the considered cooperative protocols that were analyzed by Krikidis et al. [10] is given by

$$
\begin{gathered}
C_{\mathrm{R}_{k_{-}} \mathrm{OAF}} \leq \frac{1}{2} \log _{2}\left(1+\mathrm{SNR}_{\left.\mathrm{SD}^{(1)}+\mathrm{SNR}_{\mathrm{R}_{k} \mathrm{D}}\right),}\right. \\
C_{\mathrm{R}_{k_{-}} \mathrm{NAF}} \leq \log _{2}\left\{\left(1+\mathrm{SNR}_{\mathrm{SD}}^{(1)}\right)\left(1+\mathrm{SNR}_{\mathrm{R}_{k} \mathrm{D}}+\mathrm{SNR}_{\mathrm{SD}}^{(2)}\right)\right\}, \\
C_{\mathrm{R}_{k_{-}} \mathrm{BFNAF}}^{\text {upper }}=\frac{1}{2} \log _{2}\left(1+\mathrm{SNR}_{\mathrm{SD}}^{(1)}+\mathrm{SNR}_{\mathrm{SD}}^{(2)}+\mathrm{SNR}_{\mathrm{R}_{k} \mathrm{D}}\right)
\end{gathered}
$$

where $\mathrm{SNR}_{\mathrm{SD}}^{(1)}=\gamma_{\mathrm{SD}}^{(1)}=\frac{P_{\mathrm{S}}^{(1)}}{N_{0}}\left|h_{\mathrm{SD}}^{(1)}\right|^{2}$ and $\mathrm{SNR}_{\mathrm{SD}}^{(2)}=\gamma_{\mathrm{SD}}^{(2)}=\frac{P_{\mathrm{S}}^{(2)}}{N_{0}}\left|h_{\mathrm{SD}}^{(2)}\right|^{2}$ is the instantaneous end-to-end received SNR for link $\mathrm{S} \rightarrow \mathrm{D}$ during the first phase and second phase, respectively.

To optimize the system's performance, the transmit powers of the $\mathrm{S}$ and the selected relay during both phases are fairly allocated. Hence, an efficient selection criterion for the BFNAF protocol can be obtained by maximizing the capacity of the upper bound in (11) given as

$$
\mathrm{R}_{k_{-} \mathrm{BFNAF}}=\arg \max _{k=1,2, . . K}\left\{C_{\mathrm{R}_{k_{-}} \mathrm{BFNAF}^{\mathrm{upper}}}\right\} .
$$

The summary of the new proposed RS algorithm for BFNAF protocol is given as follows:

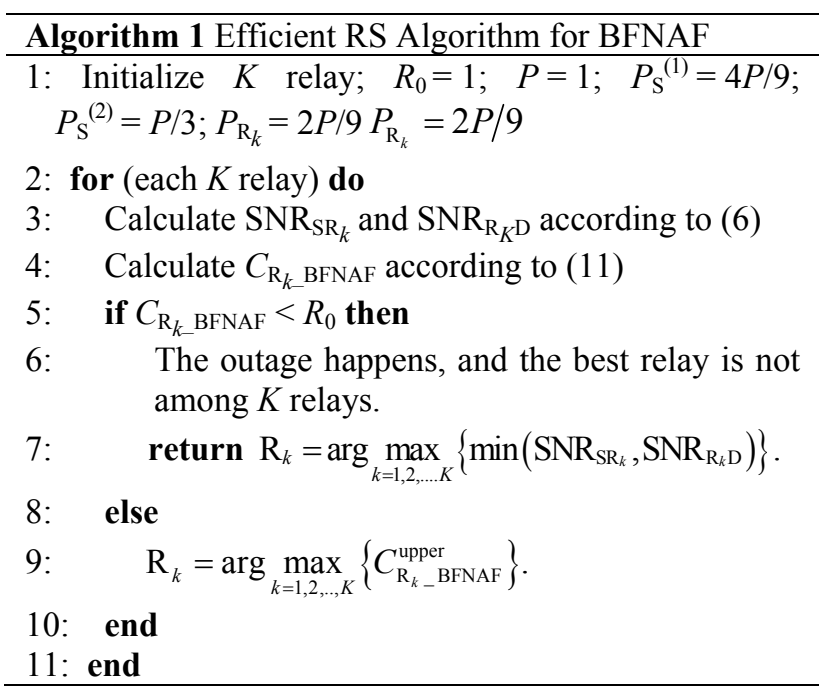

\section{Outage Probability and Throughput Analysis}

In this section, we analyze the outage probability and the throughput of the proposed RS scheme for the BFNAF cooperative networks in HD mode with optimal power allocation based on analytical expressions. The outage probability of the ORS scheme for non-orthogonal multiple-access (NOMA) with fixed power allocation has been investigated by Do \& Le in [20], in which the best relay was decided based on the end-to-end received SNR for the $\mathrm{R}_{k} \rightarrow \mathrm{D}$ link. From their observation, despite the limitation of the noise addition at the receiver, the outage performance still causes a slight degradation with the considered SNR regimes. In this work, we analyze the outage probability for the proposed RS scheme based on the channel capacity of all AF cooperative protocols, such as OAF, $\mathrm{NAF}$, and BFNAF.

We analyze the outage probability of the proposed RS scheme for the BFNAF cooperative networks in HD mode. The selection algorithm in the previous section is obtained by maximization of the capacity upper bound and still requires the optimization process. The optimization criterion is an outage probability function that corresponds to the minimum power consumption during both transmission phases. For cooperative systems, the transmit power has to be fairly allocated to all of the links and transmission phases. We assume that $P$ is the total transmission power in the communication process, and refers to the total available energy for information transmission. Generally, the outage probability is defined as the probability that the channel capacity is lower than the desired transmission rate, which is written as

$$
\begin{gathered}
\min P_{\text {out } \_ \text {coop. }}=\operatorname{Pr}\left\{C_{\mathrm{R}_{k_{-}} \text {coop. }} \leq R_{0}\right\} \\
\text { s.t }\left\{\begin{array}{c}
P_{\mathrm{S}}^{(1)}+P_{\mathrm{S}}^{(2)}+P_{\mathrm{R}_{k}}=P \\
P_{\mathrm{S}}^{(1)}, P_{\mathrm{S}}^{(2)}, P_{\mathrm{R}_{k}} \neq 0\left(\text { except } P_{\mathrm{S}}^{(2)}=0 \text { for OAF }\right) .
\end{array}\right.
\end{gathered}
$$

We also adopt the optimal power allocation policies for all cooperative schemes in [10] as the reference to optimize the transmission cost of the information signal to the destination. Krikidis et al. proposed the optimal power allocation policies based on the upper bound of capacity for the BFNAF protocol. In this work, we assume that the optimal power allocation policy for the BFNAF protocol is equal to the NAF protocol. However, the instantaneous capacity of this protocol is known for its high complexity, which makes it unable to obtain a closed-form of outage probability. Therefore, we use the capacity of the upper bound to determine the outage probability for the BFNAF protocol, which is given by

$$
\begin{aligned}
P_{\text {out_BFNAF }} & =\operatorname{Pr}\left\{C_{\mathrm{R}_{k} \_\mathrm{BFNAF}}^{\text {upper }} \leq R_{0}\right\} \\
& =\operatorname{Pr}\left\{\frac{1}{2} \log _{2}\left(1+\gamma_{\mathrm{SD}}^{(1)}+\gamma_{\mathrm{SD}}^{(2)}+\frac{\gamma_{\mathrm{SR}_{k}}^{(1)} \gamma_{\mathrm{R}_{k} \mathrm{D}}^{(2)}}{\gamma_{\mathrm{SR}_{k}}^{(1)}+\gamma_{\mathrm{R}_{k} \mathrm{D}}^{(2)}+1}\right) \leq R_{0}\right\} \\
\text { s.t } \quad P_{\mathrm{S}}^{(1)} & =\frac{4}{9} P, P_{\mathrm{S}}^{(2)}=\frac{1}{3} P, P_{\mathrm{R}_{k}}=\frac{2}{9} P .
\end{aligned}
$$

The analytical expression of the outage for the BFNAF protocol in (16) can be derived based on Lemma 1 as follows: 
Lemma 1: Let $u=\frac{4}{9}\left|h_{\mathrm{SD}}^{(1)}\right|^{2}, v=\frac{4}{9}\left|h_{\mathrm{SR}}^{(1)}\right|^{2}, w=\frac{2}{9}\left|h_{\mathrm{RD}}^{(2)}\right|^{2}$, and $z=\frac{1}{3}\left|h_{\mathrm{SD}}^{(2)}\right|^{2}$ be the independent exponential random variables, $\varepsilon=P^{-1}$, and $\lambda=\left(2^{2 R_{0}}-1\right) \varepsilon$ be the threshold of SNR. Hence, equation (14) can be written as

$$
P_{\text {out } \_ \text {BFNAF }}=\operatorname{Pr}\left\{u+z+\frac{v w}{v+w+P^{-1}} \leq\left(2^{2 R_{0}}-1\right) P^{-1}\right\} .
$$

We define $a=u+z+\frac{v w}{v+w+\varepsilon}$; thus the cumulative distribution function of $a$ can be given by

$$
\begin{aligned}
F(a) & =\operatorname{Pr}\left\{a \leq\left(2^{2 R_{0}}-1\right) \varepsilon\right\} . \\
& =1-2 \sqrt{\left(2^{2 R_{0}}-1\right) \varepsilon / u w z} K_{1}\left(\sqrt{\left(2^{2 R_{0}}-1\right) \varepsilon / u w z}\right),
\end{aligned}
$$

where $K_{1}($.$) is the Bassel function defined in [21].$ Following the calculation in [20], the outage probability in (5) can be re-written as

$$
\begin{aligned}
& P_{\text {out_BFNAF }}= \\
& 1-\left(1-e^{\frac{1}{v\left(2^{R 0}-1\right)}}\right)\left(2 \sqrt{\frac{\sigma^{2}\left(2^{R_{0}}-1\right)}{u w z P_{\mathrm{S}}^{(1)} P_{\mathrm{S}}^{(2)}}}\right) K_{1}\left(2 \sqrt{\frac{\sigma^{2}\left(2^{R_{0}}-1\right)}{u w z P_{\mathrm{S}}^{(1)} P_{\mathrm{S}}^{(2)}}}\right) .
\end{aligned}
$$

The throughput for each cooperative protocol based on the considered outage probability and threshold throughput $\tau_{\text {th }}$ can be written as

$$
\tau_{\text {BFNAF }}=\left(1-P_{\text {out_BFNAF }}\right) \tau_{\text {th }} .
$$

\section{Energy Efficiency Design}

In this section, we analyze the optimal EE design for the proposed RS scheme with different protocols (orthogonal and non-orthogonal) in HD cooperative networks. For optimal EE design, the transmit power allocation scheme is considered in [17] as the measurement of effectiveness by the normalized end-to-end received SNR at the relay node. The transmit power of the set of relays is acquired based on the maximization of their end-to-end SNR during the second phase. The combination of RS and optimal power allocation schemes based on the maximum end-to-end received SNR value at the relay node proposed in [18] can provide a better EE in both the low and high SNR regimes compared to that by using the exact SNR expression.

Motivated by these advantages, we investigated the optimal EE for the orthogonal and non-orthogonal cooperative systems of the proposed RS scheme by using the optimal power allocation for the end-to-end received SNR at the considered transmission. For the optimization process of the total EE in cooperative networks, it is necessary to calculate the total energy consumption first. The energy consumption for each transmission process is addressed in the next subsection.

\subsection{Direct Transmission}

In non-cooperative systems, the $\mathrm{S}$ directly transmits the information signal with the transmit power $P_{\mathrm{S}}=P$ to $\mathrm{D}$ without the help of relay nodes. The total energy consumption for direct transmission can be written as

$$
E_{\text {direct }}=\frac{P_{\mathrm{SD}}}{R_{\mathrm{D}}}[\text { Joule } / \text { bits }]
$$

where $P_{\mathrm{SD}}$ is the total received power of the direct transmission and $R_{\mathrm{D}}$ (in bits per second) is the data rate for the direct transmission, according to Shannon theorem [22], and can be expressed as

$$
R_{\mathrm{D}}=\frac{B}{2} \log _{2}\left(1+\mathrm{SNR}_{\mathrm{SD}}\right)
$$

\subsection{Orthogonal AF Protocol}

The EE for the OAF protocol has been proposed in [19], in which the energy consumption is calculated based on the optimal power minimization of the maximum endto-end received SNR values for the source and the selected relay node. In this paper, we calculated the energy consumption of the OAF protocol based on our proposed RS scheme along with the optimal power allocation of the transmission powers for the source and the selected relay node during the first and the second phases, which can be formulated as

$$
E_{\mathrm{OAF}}=\frac{P_{\mathrm{SD}}^{(1)}+P_{\mathrm{SR}_{k}}+P_{\mathrm{R}_{k} \mathrm{D}}}{R_{\mathrm{OAF}}}[\text { Joule/bits }]
$$

where $P_{\mathrm{SR}_{k}}$ and $P_{\mathrm{R}_{k} \mathrm{D}}$ are the received powers for the link $\mathrm{S} \rightarrow \mathrm{R}_{k}$ and $\mathrm{R}_{k} \rightarrow \mathrm{D}$ with the distances between two nodes $d_{\mathrm{SR}_{k}}$ and $d_{\mathrm{R}_{k} \mathrm{D}}$, respectively. $R_{\mathrm{OAF}}$ is the data rate for the OAF relaying system expressed as

$$
R_{\mathrm{OAF}}=\frac{B}{2} \log _{2}\left(1+\mathrm{SNR}_{\mathrm{SD}}+\mathrm{SNR}_{\mathrm{SR}_{k}}+\mathrm{SNR}_{\mathrm{R}_{k} \mathrm{D}}\right) .
$$

For the non-line of sight (NLOS) case, a direct link in the worst condition due to the poor environment or a long distance between link $\mathrm{S} \rightarrow \mathrm{D}$, the data rate in (22) can be rewritten as

$$
R_{\mathrm{OAF}}=\frac{B}{2} \log _{2}\left(1+\mathrm{SNR}_{\mathrm{SR}_{k}}+\mathrm{SNR}_{\mathrm{R}_{k} \mathrm{D}}\right) .
$$

\subsection{Block-Fading NAF Protocol}

The optimal EE design for non-orthogonal AF transmission protocol in underlay spectrum sharing systems has been proposed in [18], in which the optimization of the power allocation is obtained by the normalized end-to-end received SNR at the relay node. We adopt this scheme together with the proposed RS process for the NAF protocol by using the optimal power allocation based on the instantaneous end-to-end received SNR at the selected relay node. For the conventional NAF transmission proto- 
col with $x_{1} \neq x_{2}$ and $h_{\mathrm{SD}}{ }^{(1)}=h_{\mathrm{SD}}{ }^{(2)}$, the energy consumption for the proposed RS scheme can be obtained by

$$
E_{\mathrm{NAF}}=\frac{P_{\mathrm{SD}}^{(1)}+P_{\mathrm{SR}_{k}}^{(1)}+P_{\mathrm{SD}}^{(2)}+P_{\mathrm{R}_{k} \mathrm{D}}^{(2)}}{R_{\mathrm{NAF}}}[\text { Joule/bits] }
$$

where $R_{\mathrm{NAF}}$ (in bits per second) denotes the data rate for the NAF transmission protocol expressed as

$$
R_{\mathrm{NAF}}=\frac{B}{2} \log _{2}\left(1+\mathrm{SNR}_{\mathrm{SD}}^{(1)}+\mathrm{SNR}_{\mathrm{SD}}^{(2)}+\mathrm{SNR}_{\mathrm{SR}_{k}}+\mathrm{SNR}_{\mathrm{R}_{k} \mathrm{D}}\right)
$$

For the NLOS case, the poor channel conditions make the total end-to-end received SNRs during both direct transmissions in (25) close to zero. Thus, the data rate for the NAF protocol is equal to the data transmission rate for the OAF protocol in (23).

The EE for the BFNAF protocol has not yet been proposed in the literature. Therefore, we refer to the EE design for the conventional NAF in [17], where the optimization of the power allocation is also obtained by the normalized end-to-end received SNR at the relay node. However, due to the change of channel conditions in both transmission phases for block-fading channels, the $\mathrm{S}$ retransmits the same information signal $\left(x_{1}=x_{2}\right)$ to $\mathrm{D}$ during the second phase with different channel gains from the first phase $\left(h_{\mathrm{SD}}{ }^{(1)} \neq h_{\mathrm{SD}}{ }^{(2)}\right)$. The energy consumption for the proposed RS scheme for the BFNAF protocol along with the optimal power allocation is the same as the energy consumption for the NAF protocol in (24) and the data rate for this protocol is equal to the data rate of the NAF protocol in (25).

Furthermore, the EE for each cooperative transmission can be calculated by

$$
\begin{gathered}
\mathrm{EE}_{\mathrm{OAF}}=\frac{E_{\text {direct }}-E_{\mathrm{OAF}}}{E_{\text {direct }}} \times 100 \%, \\
\mathrm{EE}_{\mathrm{NAF}}=\mathrm{EE}_{\mathrm{BFNAF}}=\frac{E_{\text {direct }}-E_{\mathrm{NAF}}}{E_{\text {direct }}} \times 100 \% .
\end{gathered}
$$

\section{Numerical Results}

In this section, we present the numerical results of the channel capacity bounds for each protocol, outage and throughput performance, and the EE for the proposed RS scheme with the OAF, NAF, and BFNAF protocols for HD cooperative networks. The Monte Carlo simulations of numerical analysis were carried out by using MATLAB R2015b release over a Rayleigh block-flat fading channel. All channel coefficients of the block-flat fading model are assumed to be independent and invariant during both transmission phases $\left(h_{\mathrm{SD}}{ }^{(1)} \neq h_{\mathrm{SD}}{ }^{(2)}\right)$, in which the $\mathrm{S}$ continuously maintains the transmission of the same data $\left(x_{1}=x_{2}\right)$ while $\mathrm{R}_{k}$ retransmits the amplified version of the data signal from the source node.

Two different modulation schemes are considered in this paper, i.e. BPSK for outage performance and throughput and BPSK/16-QAM for the EE comparison. The parameters are considered for the outdoor $4 \mathrm{G}$ wireless

\begin{tabular}{|l|c|}
\hline \multicolumn{1}{|c|}{ Parameter } & Parameter value \\
\hline Channel bandwidth, $B$ & $200 \mathrm{MHz}$ \\
\hline Operation frequency, $f$ & $2.3-2.5 \mathrm{GHz}$ \\
\hline Total transmission power, $P$ & $250 \mathrm{~mW} / 24 \mathrm{dBm}$ \\
\hline Path-loss exponent, $\alpha$ & 2 \\
\hline Thermal noise power spectral density, $N_{0}$ & 1 \\
\hline Noise variances, $\sigma_{\mathrm{S}}{ }^{2}=\sigma_{\mathrm{R}}{ }^{2}=\sigma_{\mathrm{D}}{ }^{2}=\sigma^{2}$ & 1 \\
\hline Threshold rate, $R_{0}$ & $1 \mathrm{bit} / \mathrm{s} / \mathrm{Hz}$ \\
\hline No. of information bits & $10^{6} \mathrm{bits}$ \\
\hline Threshold throughput & $1 \mathrm{Gbps}$ \\
\hline Distance ratio between $\mathrm{S}-\mathrm{R}_{k}, \mathrm{R}_{k}-\mathrm{D}, d_{\mathrm{RD}}, d_{\mathrm{SR}}$ & $0.1-0.9$ \\
\hline Modulation scheme & $\mathrm{BPSK}, 16-\mathrm{QAM}$ \\
\hline No. of relays, $K$ & $1-4$ \\
\hline SNR regime & $0-30 \mathrm{~dB}$ \\
\hline Average time consumption of simulation & $190.8 \mathrm{~s}$ \\
\hline
\end{tabular}

Tab. 1. Parameter setup.

technology with channel bandwidth $B=200 \mathrm{MHz}$ and working frequency range $2.3-2.5 \mathrm{GHz}$. The transmit power for the mobile-user is equal to $P=250 \mathrm{~mW}$ or $24 \mathrm{dBm}$ and the number of information bits is assumed as $N=10^{6}$. The distance ratio between links $\mathrm{S} \rightarrow \mathrm{D}, \mathrm{S} \rightarrow \mathrm{R}_{k}$, and $\mathrm{R}_{\mathrm{k}} \rightarrow \mathrm{D}$ is varied to $0-1,0.1-0.9$, and $0.9-0.1$, respectively. We assumed that the threshold rate is equal to $R_{0}=1 \mathrm{bit} / \mathrm{s} / \mathrm{Hz}$ and the threshold SNR given by $\lambda=\left(2^{2 R_{0}}-1\right) \varepsilon$ with $\varepsilon=P^{-1}$. In particular, the parameters set up are given in Tab. 1 . The proposed simulation model of this paper can be found at the author's Research Gate profile ${ }^{1}$.

\subsection{Capacity Bounds}

The capacity bounds for the considered cooperative transmissions, i.e. the OAF, the NAF, and the BFNAF protocols, have been analyzed in Sec. 3 based on (9), (10), and (11), respectively. In this section, we compared the capacity bounds of these three protocols for two different power allocation policies to determine the best policy to be used in the next simulations. For the first policy, we assumed that the transmission power for $\mathrm{S}$ and the selected relay $\mathrm{R}_{k}$ during both transmission phases were equal for both non-orthogonal and orthogonal protocols. For the second policy, the orthogonal and non-orthogonal protocols had a different power allocation for each node during both transmission phases.

The comparison of the channel capacities of the three cooperative transmission protocols for the two power allocation policies is illustrated in Fig. 2. More specifically, we compared the Shannon capacity for the direct link, the $\mathrm{OAF}, \mathrm{NAF}$, and BFNAF relaying protocols with the symmetric power allocation policy, i.e., for the BFNAF and NAF $\left(P_{\mathrm{S}}^{(1)}=P_{\mathrm{S}}^{(2)}=P_{\mathrm{R}_{k}}=P / 3\right)$ and the OAF $\left(P_{\mathrm{S}}^{(1)}=P_{\mathrm{R}_{k}}=P / 2\right)$; and the optimal power allocation, i.e., for the BFNAF and NAF $\left(P_{\mathrm{S}}^{(1)}=4 P / 9, P_{\mathrm{S}}^{(2)}=P / 3\right.$, and $\left.P_{\mathrm{R}_{k}}=2 P / 9\right)$ and the OAF $\left(P_{\mathrm{S}}^{(1)}=2 P / 3, P_{\mathrm{R}_{k}}=P / 3\right)$.

We assumed that the transmission power for each node during the first and the second phase is equal to the

\footnotetext{
${ }^{1}$ https://www.researchgate.net/profile/Yunida Yunida
} 


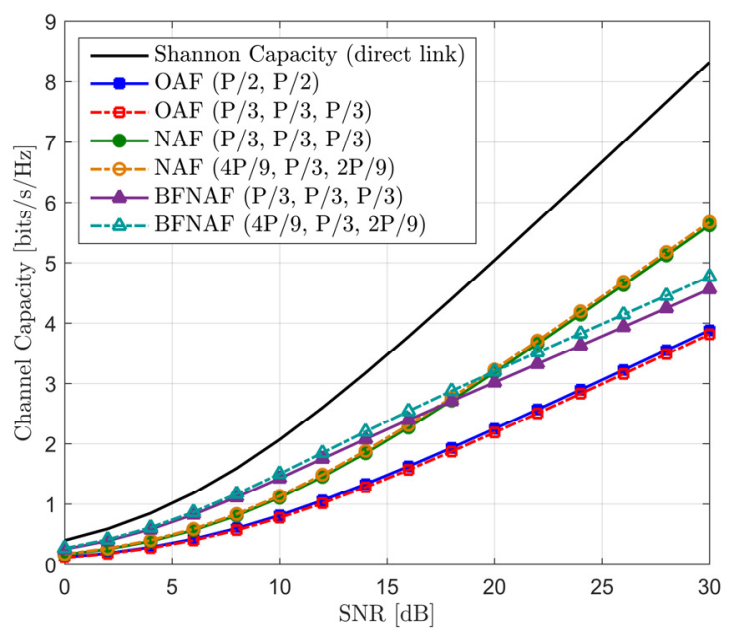

Fig. 2. Channel capacities comparison for orthogonal and non-orthogonal protocols with different power allocation policies.

linear SNR value. The comparison of power allocation policies shows that the optimal power allocation policy provided a slightly better channel capacity than that of the symmetric power allocation. From the illustration, it can also be observed that the BFNAF protocol significantly outperforms both the OAF and the NAF protocol in the lower SNR regime (0-18 dB) while the NAF outperforms both the OAF and the BFNAF in the higher SNR regime $(\geq 18 \mathrm{~dB})$.

At a higher SNR regime, the capacity gains for the NAF protocol compared to those of the BFNAF and the $\mathrm{OAF}$ are respectively equal to $1 \mathrm{~dB}$ and $2 \mathrm{~dB}$. It can be concluded that the non-orthogonal protocols provide a better capacity than the orthogonal protocol. This result proves that the inactivity of the source node during the second phase $\left(P_{\mathrm{S}}^{(2)}=0\right)$ for the OAF protocol causes a loss of the transmission rate. Hence, the optimal power allocation policy is that in which the transmission powers are differently allocated for the optimal EE purpose.

\subsection{Outage and Throughput Performance}

The outage performance results of the proposed RS scheme for non-cooperation, the OAF, NAF, and BFNAF protocol are shown in Fig. 3. We varied the numbers of relay nodes from 1 to 4 , using the BPSK modulation scheme with the optimal power allocation policies over the block-fading channel. The simulation result shows the outage probability $\left(P_{\text {out }}\right)$ comparison of the direct link, BFNAF, and OAF protocol of the proposed relay selection for different numbers of relay nodes, $K=1,2,3,4$. Based on the simulation result, we can see that the outage probability of the BFNAF protocol with the proposed RS scheme provides a better performance gain along with the increase of the number of relay nodes $K$.

For example, at a $10^{-6}$ outage probability, it can be observed that when the numbers of relays are increased from $K=2$ to $K=4$, the performance gain obtained is approximately $2.5 \mathrm{~dB}$ at low SNR regimes $(0-14 \mathrm{~dB})$. On the other

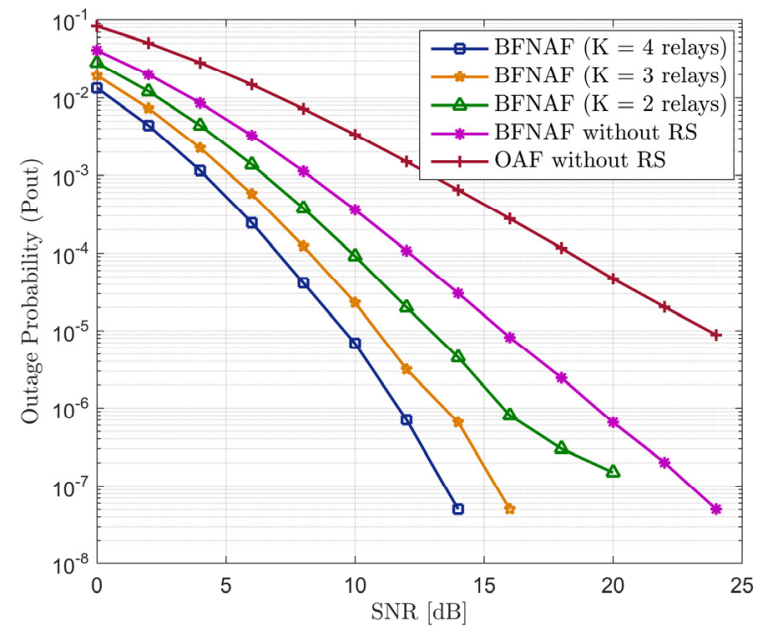

Fig. 3. Outage performance of the proposed RS scheme for the BFNAF protocol using BPSK modulation with different numbers of relay nodes $(K)$.

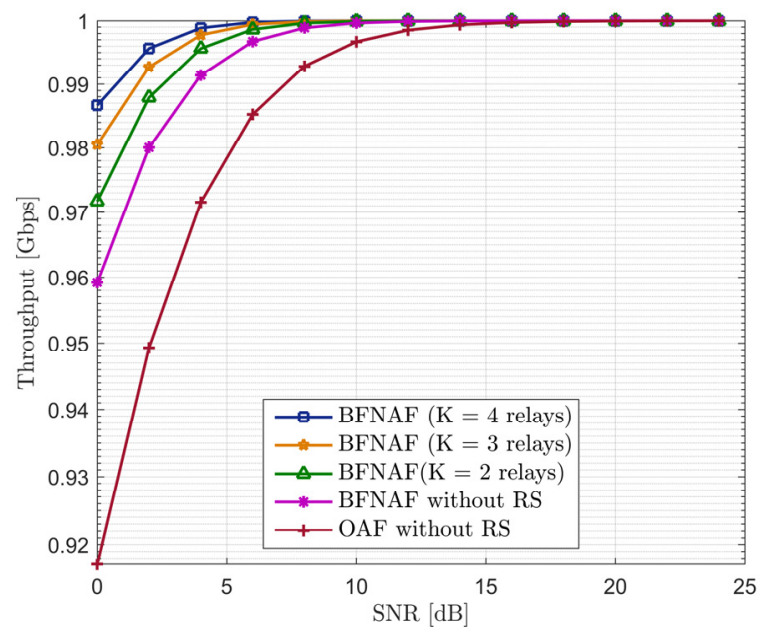

Fig. 4. Throughput of the proposed RS scheme for the BFNAF protocol using BPSK modulation with different numbers of relay nodes $(K)$.

hand, for high SNR regimes $((\geq 14 \mathrm{~dB})$, the outage provides a greater performance gain, especially for $K=4$. This indicates that the outage performances for the BFNAF protocol with the proposed RS scheme can be improved not only by adding more relay nodes but also by increasing the transmit SNR.

Next, we observe the throughput for the BFNAF protocol using the proposed RS with a different number of relays as illustrated in Fig. 4. Contrary to the outage performance, the overall performance shows that the BFNAF with the proposed RS scheme provides a higher throughput by increasing the transmit SNR and the number of relays. Based on the illustration, the BFNAF with $K=4$ relays is significantly approaching the data rate threshold $\left(R_{0}=\right.$ $1 \mathrm{bit} / \mathrm{s} / \mathrm{Hz}$ ).

The outage performance comparison of the proposed RS scheme for the BFNAF and the OAF protocols with different power allocation policies for $K=4$ is shown in Fig. 5. More specifically, for the first policy, we assumed that all nodes have equal or symmetric power allocation for 


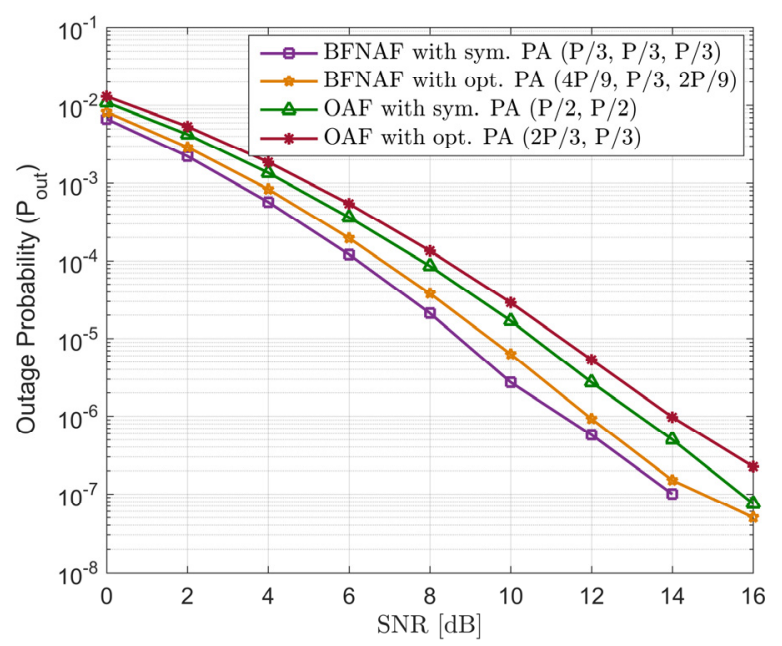

Fig. 5. Outage performance of the proposed RS scheme for BFNAF and OAF protocols using BPSK modulation with different power allocation (PA) policies and $K=4$.

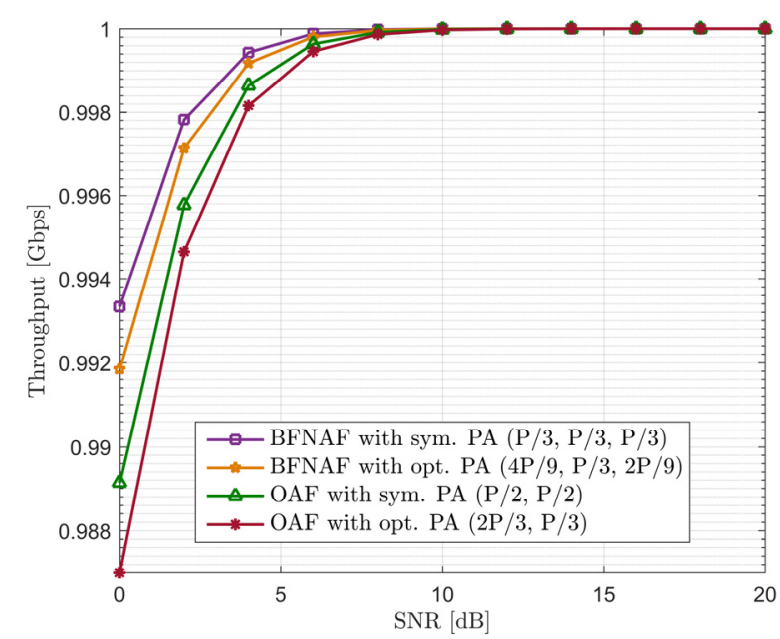

Fig. 6. Throughput of the proposed RS scheme for BFNAF and OAF protocols using BPSK modulation with different power allocation (PA) policies and $K=4$.

both the OAF and BFNAF protocol. On the other hand, the second policy is the optimal power allocation policy with different transmit power during both transmission phases for each node. From the illustration, we can see that the proposed RS scheme with the BFNAF protocol outperforms the OAF protocol in both symmetric and optimal PAs.

Specifically, the optimal PA provides a better outage performance gain compared to the symmetric PA with the diversity gain up to $0.5 \mathrm{~dB}$ in the high $\mathrm{SNR}$ regime. This indicates that the optimal PA can dominate the power constraint, which is related to the end-to-end SNR value at the relay node. Thus, we conclude that the proposed RS scheme for the BFNAF protocol with optimal PA can obtain a better diversity gain compared to the OAF protocol because there is an additional transmission by a source to the destination during the second phase. Furthermore, for the OAF protocol, the maximum amplification factor cannot compensate the result of the amplified noise signal at the destination and fails to increase the performance gain due to the inactive source node in the second transmission phase.

In addition to the power allocation, we also compared the throughput of the proposed RS scheme for the BFNAF and OAF protocols with symmetric and optimal power allocation, as shown in Fig. 6. It is shown that the BFNAF protocol with the optimal power allocation provides higher throughput than other schemes along with increasing the transmit SNR. In the case of the symmetric power allocation, the BFNAF also provides a better throughput compared to the OAF protocol. Thus, we can conclude that the improvement of the throughput for the BFNAF protocol is obtained by the optimal power allocation policy and by increasing the transmit power at the relay and source nodes.

\subsection{Energy Efficiency}

In this subsection, we provide the optimized EE performance under the proposed RS algorithm for the BFNAF and the OAF protocols and compare it to the ORS scheme with a number of relays $K=3$. The total energy consumptions for both protocols are calculated with the given optimal power allocation during both transmission phases. The EE performance comparisons were performed based on improvement of the distance ratio between the two nodes $\left(\mathrm{S} \rightarrow \mathrm{R}_{k}\right.$ and $\left.\mathrm{R}_{k} \rightarrow \mathrm{D}\right)$ by using BPSK and 16-QAM modulation, depicted in Fig. 7 and Fig. 8, respectively.

By observing these two results, we can see that the BFNAF protocol with the proposed RS algorithm achieves the highest optimized EE among the other schemes for both modulation schemes. As an example, at the same distance ratio of 0.1 , the optimized EE by the proposed RS with the BFNAF protocol for the 16-QAM can be up to $85.80 \%$ higher than that of the BPSK, with only $36.9 \%$. Moreover, for the BPSK scheme, the OAF protocol with the proposed RS achieves a higher optimized EE compared to the BFNAF with the ORS scheme, with a difference of $18.9 \%$. Meanwhile, for the 16-QAM scheme, the BFNAF protocol with the ORS scheme provides a higher optimized EE compared to the OAF protocol with the proposed RS, with a difference of $11.02 \%$.

Based on the analysis of the proposed RS scheme, the relay selected is the one with a maximum capacity bound that is equal to the maximum end-to-end received SNR at the relay node. Therefore, the optimized EE under the proposed RS scheme automatically depends on the distance ratio between $\mathrm{R}_{k} \rightarrow \mathrm{D}$. In other words, the farther the distances between the selected relay and source, the greater the transmit power needed to send the information signal to the destination.

Furthermore, it can be concluded that the proposed RS algorithm with the BFNAF protocol can reduce the total energy consumption at the destination by the selection of a cooperating node to forward the information from $S$ to the $\mathrm{D}$ with the minimum overall energy provided in the communication process. Due to the complexity of the analysis of the outage probability using $M$-QAM, we decided to 


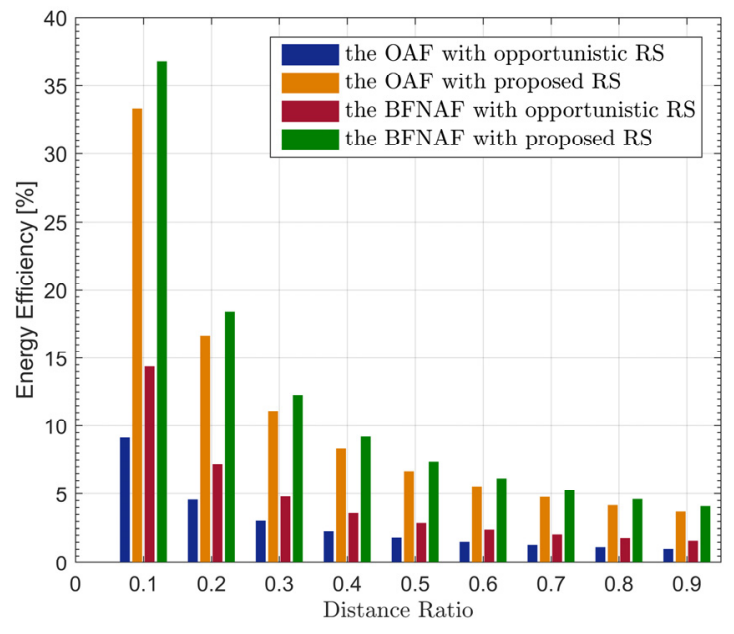

Fig. 7. Optimized EE for the BFNAF and OAF protocol in HD cooperative networks using BPSK modulation.

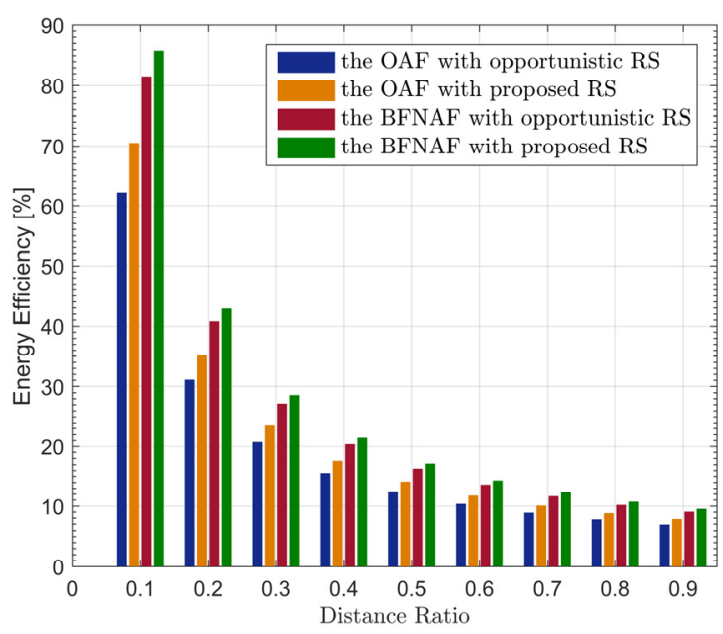

Fig. 8. Optimized EE for the BFNAF and OAF protocol in HD cooperative networks using 16-QAM modulation.

only use BPSK modulation for outage performance and throughput in this work. For further research, it is better to consider the $M$-QAM modulation for outage probability and throughput analysis in order to increase the performance gain of cooperative networks.

\section{Conclusions}

In this paper, we have proposed an efficient relay selection for HD cooperative networks with the BFNAF relaying protocol. The system consists of a single source node that wants to communicate with a destination by using the help of several relays. A new relay selection algorithm is proposed to optimize the EE using the maximum capacity bound criterion of the link between relays and destination under the considered transmission power. We have also provided the outage probability and throughput analysis as the performance parameters for the BFNAF protocol with the proposed RS scheme. Then, the EE is designed to determine the energy consumption for the OAF and BFNAF protocol under the optimized transmission power allocation. Moreover, computer simulations have been done to observe the diversity gain and the optimized EE performance for each cooperative protocol. The simulation results show that our proposed RS scheme with the BFNAF protocol provides a better outage performance and throughput together with the addition of a power allocation policy compared to those of the ORS and the OAF protocol. The throughput can also be improved by increasing the number of relays and by allocating power constraints at the relay node. Based on the EE comparison, the average optimized EE of the proposed RS algorithm with the BFNAF protocol is up to $85.80 \%$ higher than that of the ORS scheme with the OAF protocol in previous work, with only $62.60 \%$ for $16-\mathrm{QAM}$.

\section{Acknowledgments}

This work was supported by a scholarship from the "Magister Program of Education Leading to Doctoral for Excellent Graduates", the Ministry of Education and Culture of the Republic of Indonesia.

\section{References}

[1] SENDONARIS, A., ERKIP, E., AAZHANG, B. User cooperation diversity. Part II: Implementation aspects and performance analysis. IEEE Transactions on Communication, 2003, vol. 51, no. 11, p. 1939-1948. DOI: 10.1109/TCOMM.2003.819238

[2] LANEMAN, J. N., TSE, D. N. C., WORNELL, G. Cooperative diversity in wireless networks: Efficient protocols and outage behaviour. IEEE Transactions on Information Theory, 2004, vol. 50, no. 12, p. 3062-3080. DOI: 10.1109/TIT.2004.838089

[3] LI, E., WANG, X., WU, Z., et al. Outage analysis of decode-andforward two-way relay selection with different coding and decoding schemes. IEEE Systems Journal, 2019, vol. 13, no. 1, p. 125-136. DOI: 10.1109/JSYST.2018.2810019

[4] ZHAO, D., ZHAO, H., JIA, M., et al. Smart relaying for selection combining based decode-and-forward cooperative networks. IEEE Communications Letters, 2014, vol. 18, no. 1, p. 74-77. DOI: 10.1109/LCOMM.2013.112513.132216

[5] LEE, I. H. Outage performance of efficient partial relay selection in amplify-and-forward relaying systems over Rayleigh fading channels. IEEE Communications Letters, 2012, vol. 16, no. 10, p. 1644-1647. DOI: 10.1109/LCOMM.2012.090312.121523

[6] LEE, J., RIM, M., KIM, K. On the outage performance of selection amplify-and-forward relaying scheme. IEEE Communications Letters, 2014, vol. 18, no. 3, p. 423-426. DOI: 10.1109/LCOMM.2014.011214.132477

[7] NGUYEN, K. T., DO, D. T., VOZNAK, M. An optimal analysis in wireless powered full-duplex relaying network. Radioengineering, 2017, vol. 26, no. 1, p. 369-375. DOI: 10.13164/re.2017.0369

[8] NABAR, R. U., BÖLCSKEI, H., KNEUBUHLER, F. W. Fading relay channel: Performance limits and space-time signal design. IEEE Journal on Selected Areas in Communication, 2004, vol. 22, no. 6, p. 1099-1109. DOI: 10.1109/JSAC.2004.830922

[9] ELSAADANY, M., HAMOUDA, W. Performance analysis of non-orthogonal AF relaying in cognitive radio networks. IEEE Wireless Communications Letters, 2015, vol. 4, no. 4, p. 373-376. DOI: 10.1109/LWC.2015.2421910 
[10] KRIKIDIS, I., THOMPSON, J., MCLAUGHLIN, S., et al. Optimization issues for cooperative amplify-and-forward systems over block-fading channels. IEEE Transaction on Vehicle Technology, 2008, vol. 57, no. 5, p. 2868-2884. DOI: 10.1109/TVT.2008.917235

[11] YUNIDA, Y., NASARUDDIN, N., MUHARAR, R., et al. Linear precoder design for non-orthogonal AF MIMO relaying systems based MMSE criterion. EURASIP Journal on Wireless Communication and Networking, 2018, vol. 2018, p. 1-8. DOI: 10.1186/s13638-018-1295-y

[12] YUNIDA, Y., NASARUDDIN, N., MUHARAR, R., et al. Implementation of the Alamouti STBC for multi-pair two-way wireless networks with amplify-and-forward MIMO relaying. In Proceedings of IEEE International Conference on Information Technology Systems and Innovation (ICITSI). Bandung-Padang (Indonesia), 2018, p. $\quad 515-519 . \quad$ DOI: 10.1109/ICITSI.2018.8695941

[13] BLETSAS, A., KHISTI, A., REED, D., et al. A simple cooperative diversity method based on network path selection. IEEE Journal on Selected Areas in Communication, 2006, vol. 24, no. 3, p. 659-672. DOI: 10.1109/JSAC.2005.862417

[14] LI, D. Opportunistic DF-AF selection for cognitive relay networks. IEEE Transactions on Vehicular Technology, 2016, vol. 65 , no. 4. p. 2790-2796. DOI: 10.1109/TVT.2015.2418535

[15] KRIKIDIS, I., SURAWEERA, H. A., SMITH, P. J., et al. Fullduplex relay selection for amplify-and-forward cooperative networks. IEEE Transactions on Wireless Communication, 2012, vol. 11, no. 12, p. 4381-4393, DOI: 10.1109/TWC.2012.101912.111944

[16] YIN, C., DOAN, T. X., NGUYEN, N. P., et al. Outage probability of full-duplex cognitive relay networks with partial relay selection. In Proceedings of IEEE International Conference on Recent Advance in Signal Processing, Telecommunication \& Computing. Da Nang (Vietnam), 2017, p. 115-118. DOI: 10.1109/SIGTELCOM.2017.7849806

[17] ELSAADANY, M., HAMOUDA, W. Energy-efficient design for non-orthogonal AF relaying in underlay spectrum sharing networks. In Proceedings of IEEE International Conference on Communication. Kuala Lumpur (Malaysia), 2016, p. 1-6. DOI: 10.1109/ICC.2016.7510859

[18] KHALIL, M. I., BERBER, S. M., SOWERBY, K. W. Energy efficiency design for combined relay selection and power allocation AF-relay network. In Proceedings of The 20th IEEE Asia-Pacific Conference on Communication (APCC). Pattaya (Thailand), 2014, p. 417-422. DOI: 10.1109/APCC.2014.7092848

[19] HWANG, K. S., KO, Y. C. An efficient relay selection algorithm for cooperative networks. In Proceedings of the IEEE $66^{\text {th }}$ Vehicle Technology Conference. Baltimore (USA), 2007, p. 81-85. DOI: 10.1109/VETECF.2007.33

[20] DO, D. T., LE, A. T. NOMA based cognitive relaying: Transceiver hardware impairments, relay selection policies and outage performance comparison. Journal of Computer Communication, 2019, vol. 146, p. 144-154. DOI: 10.1016/j.comcom.2019.07.023

[21] DAVID, H. A. Order Statistic. 1st ed. New York, NY (USA): Wiley, 1970.

[22] SHANNON, C. E. Communication in the presence of noise. Proceedings of IEEE, 1984, vol. 72, no. 9, p. 1192-1201. DOI: 10.1109/PROC.1984.12998

\section{About the Authors ...}

Yunida YUNIDA was born in 1991 in Southwest Aceh, Indonesia. She received B. Eng. degree in Electrical Engineering from Syiah Kuala University, Indonesia in 2013. She is currently a master toward a doctoral student in the Electrical and Computer Engineering Department, Syiah Kuala University. She is also a research assistant in Telecommunication Laboratory. Her research interests include coding and signal processing for wireless cooperative communication networks.

Rusdha MUHARAR obtained the Bachelor of Engineering degree from Gadjah Mada University, Indonesia, in 1999, the M.Sc. degree from TU Delft, The Netherlands, in 2004 and the Ph.D. degree from the University of Melbourne, Australia, in 2012, all in Electrical Engineering. He was a post-doctoral research fellow at Dept. Electrical \& Computer System Engineering, Monash University from Nov. 2012 to Nov. 2013. Currently, he is the lecturer at the Department of Electrical and Computer Engineering, Syiah Kuala University. His research interests are in wireless communications, information theory, and signal processing $\&$ its applications.

Yuwaldi AWAY obtained his degree in Electrical-Computer Engineering (1988) from Sepuluh Nopember Institute of Technology (ITS), Indonesia. He starts his carrier as a lecturer in 1990 at Syiah Kuala University, Indonesia (1990-now). He earned his M.Sc. (1993) from Bandung Institute of Technology (ITB), Indonesia, and he finished his Ph.D. (2000) in an Industrial Computer from the National University of Malaysia. From 1996 to 2004, he was a lecturer as well as a research assistant at the National University of Malaysia. Now he is a professor (2007-now) at Syiah Kuala University. The research scope is a combination of theory and practical, including the microprocessor, simulation, automation, and optimization.

Nasaruddin NASARUDDIN (Corresponding author) received the B.Eng. degree in Electrical Engineering from Sepuluh Nopember Institute of Technology, Surabaya, Indonesia in 1997. Then he received M. Eng. and D. Eng. in Physical Electronics and Informatics, Graduate School of Engineering, Osaka City University, Japan, in 2006 and 2009 , respectively. He is a full professor at the Electrical and Computer Engineering Department, Syiah Kuala University. He was head of master of Electrical Engineering Programme; graduate school of Syiah Kuala University. Currently, he is head of the Electrical and Computer Engineering Department, Faculty of Engineering, Syiah Kuala University. His research interests include digital communications, information theory, computer, and communications networks. 